\title{
Pythiosis mamaria en una yegua criolla colombiana
}

\author{
Mammary pythiosis in a Colombian creole mare
}

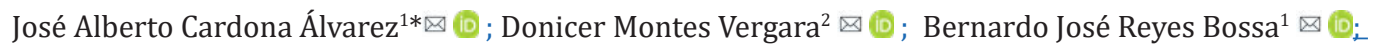

\begin{abstract}
${ }^{1}$ Universidad de Córdoba, Facultad de Medicina Veterinaria y Zootecnia, Departamento de Ciencias Pecuarias, Grupo de Investigaciones en Medicina de Grandes Animales (MEGA), Montería, Colombia.

${ }^{2}$ Universidad de Sucre. Facultad de Ciencias Agropecuarias, Departamento de Zootecnia. Sincelejo, Colombia.

*Correspondencia: jacardonaalvarez@correo.unicordoba.edu.co
\end{abstract}

Recepción: 18 abril 2021 | Aprobación: 23 junio 2021 | Publicación: 5 agosto 2021

\begin{abstract}
RESUMEN
Se reporta un caso de una yegua criolla colombiana, color castaño, de 7 años y un peso de $300 \mathrm{Kg}$, la cual fue atendida por el Servicio Clínico Ambulatorio del área de Clínica Médico-Quirúrgica de Grandes Animales de la Universidad de Córdoba. La anamnesis indica que la yegua presentó un aumento progresivo de tamaño a nivel de la glándula mamaria, con secreción mucopurulenta constante por diferentes orificios, así mismo el propietario reporta que le realizó incisiones para facilitar el drenaje de material desde la glándula mamaría, ya que pensaba que ese aumento consistía en un absceso, de igual forma, informó la aplicación de antibióticos a base de tetraciclinas y antiinflamatorios no esteroidales. Al examen clínico, se determinó la presencia de una lesión granulomatosa que comprometía toda la glándula mamaria, con presencia de varias aberturas en forma de cráter y salida de material fibrinopurulento, a la compresión presento salida de mayor cantidad de secreción, así como masas caseificadas llamadas Kunker. Se decidió realizar mastectomía total como medida de control y alargar la vida útil de la yegua. Durante el procedimiento quirúrgico bajo anestesia general, se evidenció la presencia de abundante tejido granulomatoso, así como la presencia de grandes cantidades de kunkers acumulados en cavitaciones originadas por la invasión del Pythium insidiosum en el tejido granular. Se concluye la importancia de conocer la epidemiología de la Pythiosis, siendo relevante la ubicación de casos clínicos a nivel de glándula mamaria en yeguas criollas colombianas expuestas a factores de riesgo.
\end{abstract}

Palabras clave: Pythium insidiosum; mastitis; oomiceto; equino.

\begin{abstract}
A case is reported of a Colombian Creole mare, brown, 7 years old and weighing $300 \mathrm{Kg}$, which was treated by the Outpatient Clinical Service of the Large Animal Medical-Surgical Clinic area of the University of Córdoba. The anamnesis indicates that the mare presented a progressive increase in size at the level of the mammary gland, with constant mucopurulent discharge from different orifices, likewise the owner reports that he made incisions to facilitate the drainage of material from the mammary gland, since he thought that this increase consisted of an abscess, in the same way, the application of antibiotics based on tetracyclines and non-steroidal anti-inflammatory drugs reported. At the clinical examination, the presence of a granulomatous lesion that compromised the entire mammary gland was determined, with the presence of several crater-shaped openings and exit of fibrinopurulent material, upon compression I presented an exit of a greater amount of secretion, as well as caseified masses called Kunker. It was decided to perform a total mastectomy as a control measure and to extend the life of the mare. During the surgical procedure under general anesthesia, the presence of abundant granulomatous tissue was evidenced, as well as the presence of large amounts of kunkers accumulated in cavities caused by the invasion of Pythium insidiosum in the granular tissue. The importance of knowing the epidemiology of Pythiosis is concluded, being relevant the location of clinical cases at the mammary gland level in Colombian Creole mares exposed to risk factors.
\end{abstract}

Keywords: Pythium insidiosum; mastitis; oomycete; equine. 


\section{INTRODUCCIÓN}

La pythiosis cutánea equina es una enfermedad de la piel de los equinos, que pastan en regiones tropicales y subtropicales con presencia de zonas inundables. Es causada por el Oomiceto Pythium insidiosum $(1,2)$

El Pythium insidiosum es un microorganismo clasificado como perteneciente al reino Stramenopila, phylum Pseudofúngi, clase Oomycetes, orden Pythiales, familia Pythiaceae y género Pythium, de modo que los miembros de la clase Oomycetes son filogenéticamente distantes del reino de los hongos y más cercanos de las algas (3).

Las condiciones ambientales son determinantes para el desarrollo del microorganismo en su ecosistema. Para haber la producción de zoosporas son necesarias temperaturas entre 30 y $40^{\circ} \mathrm{C}$ y el acúmulo de agua en potreros o represas. La gran mayoría de los casos de pythiosis ha sido observada durante o después de la época lluviosa $(4,5)$.

Las lesiones se caracterizan por su apariencia granulomatosa y en forma de cráter, con presencia de prurito, trayectos fistulosos, secreción fibrino-sanguinolenta y salida de material necrótico, caseificado llamado "kunkers" $(2,6)$.

Los sitios de ubicación más comunes de lesiones por pythiosis son principalmente las partes distales de los miembros, seguida de barbilla, abdomen y pecho respectivamente; siendo atribuido al hecho de que estas áreas sean de mayor exposición al Oomiceto, por ser de mayor contacto con aguas contaminadas (5). Razón por la cual, el objetivo del presente reporte es dar a conocer una ubicación no habitual en la presentación de la casuística clínica de la pythiosis cutánea en equinos del trópico bajo colombiano.

\section{DESCRIPCIÓN DEL CASO}

Antecedentes. Fue atendido por el Servicio Clínico Ambulatorio de Grandes Animales de la Universidad de Córdoba, una yegua criolla colombiana, color castaño, de 7 años y un peso de $300 \mathrm{Kg}$, ubicado en la vereda manguelito del Municipio de Cereté, Córdoba.

Anamnesis. El encargado del manejo de la pesebrera, reportó que la yegua presentó un aumento progresivo de tamaño a nivel de la glándula mamaria, con secreción mucopurulenta constante por diferentes orificios, así mismo informó que le realizó incisiones para facilitar el drenaje de material desde la glándula mamaría, ya que pensaba que ese aumento consistía inicialmente en una picadura de araña violinista y posteriormente un absceso, de igual forma, informó la aplicación de antibióticos a base de tetraciclinas y antiinflamatorios no esteroidales, sin encontrar mejoría aparente.

Examen Físico. A la inspección, se determinó la presencia de una lesión granulomatosa que comprometía toda la glándula mamaria, con presencia de varias aberturas en forma de cráter y salida de material fibrinopurulento (Figura 1).

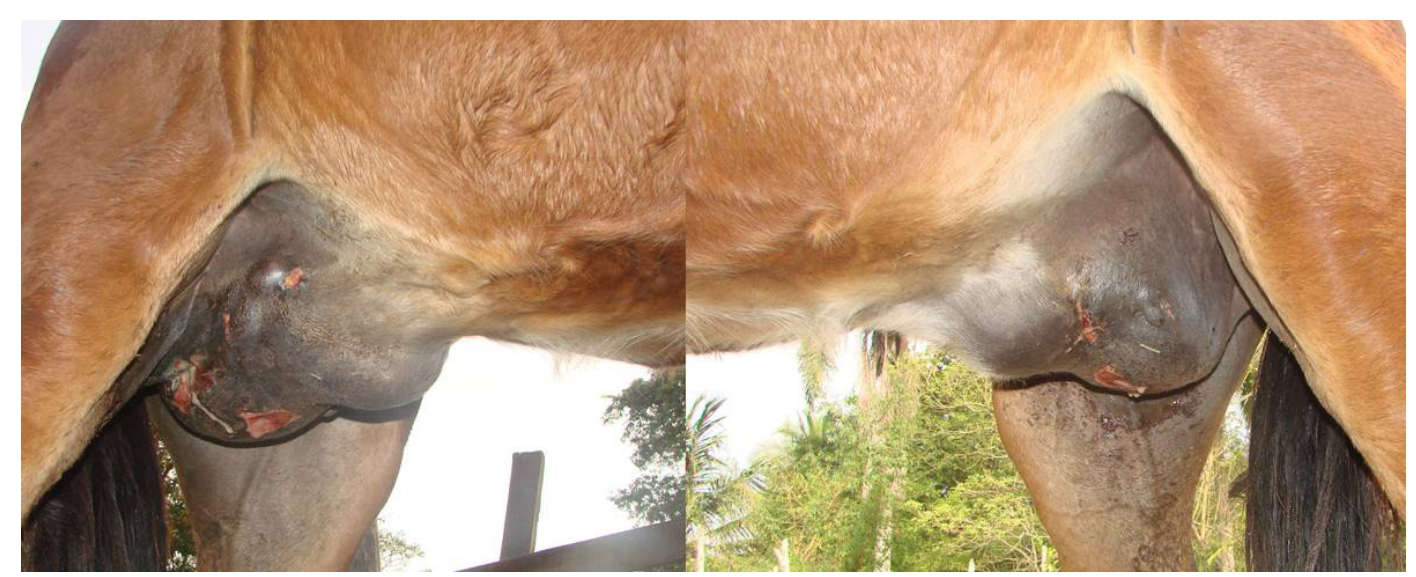

Figura 2. Glándula mamaria. Observe el aumento de volumen de la glándula mamaria, en su vista lateral derecha e izquierda respectivamente.

A la palpación, se estableció que la masa comprometía todo el parénquima glandular, siendo de consistencia dura, a la compresión presentó salida de mayor cantidad de secreción, así como masas caseificadas llamadas Kunker. (Figura 2). 


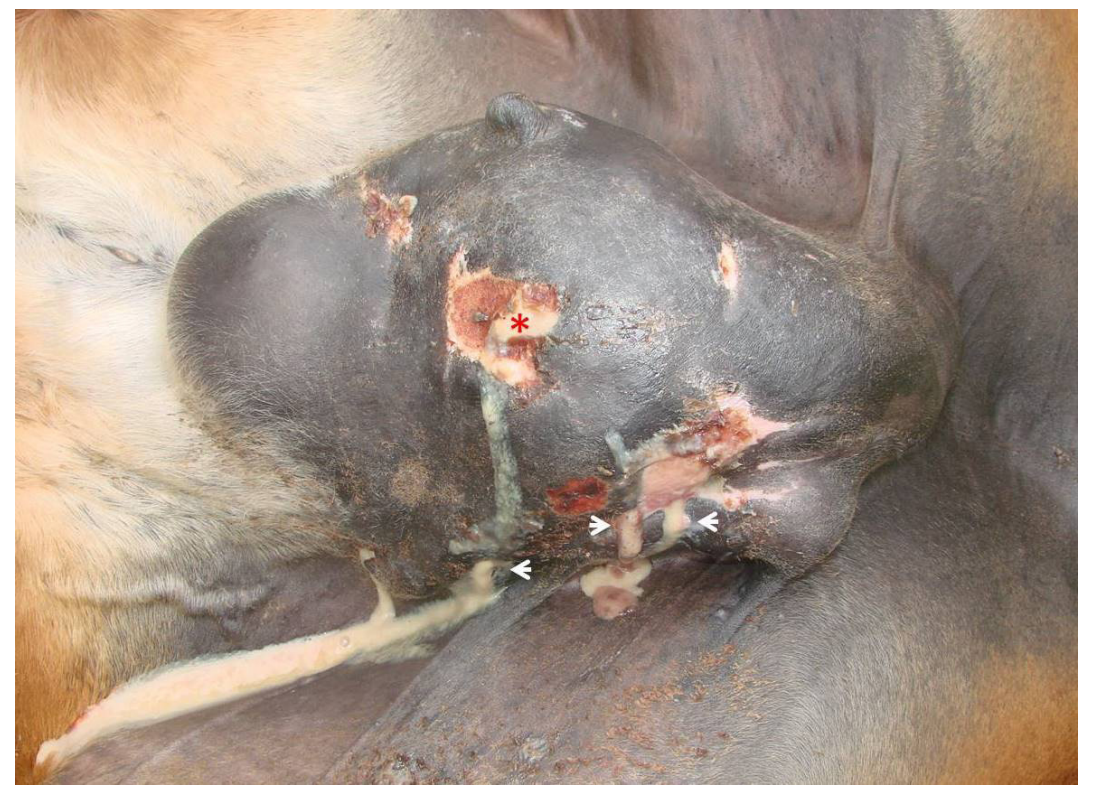

Figura 2. Mastitis granulomatosa. Observe la presencia de una lesión granulomatosa que comprometía toda la glándula mamaria, con presencia de varias aberturas en forma de cráter (asterisco) y salida de material fibrinopurulento por diferentes partes (cabeza de flecha).

Diagnóstico. El diagnóstico inicial de mastitis por Pythium insidiosum se realizó por las características clínicas y anatomopatológicas de la lesión según lo reportado por Cardona (7) y Cardona et al $(2,3,6)$. Así mismo fue colectada biopsia mediante punch de $6 \mathrm{~mm}$ y conservada en formalina tamponada al 10\% hasta su procesamiento y confirmación por histopatología en las coloraciones de Hematoxilina Eosina (HE) y Grocott (GSM) $(8,9)$.

Procedimiento. Teniendo en cuenta la notable gravedad de la condición debido a los procesos cicatrizales de la piel producto de las incisiones realizadas con anterioridad, se decidió realizar mastectomía total como medida de control y alargar la vida útil de la yegua.

Para realizar el procedimiento quirúrgico en el animal se tuvieron en cuenta las normas técnicas referentes a los principios éticos internacionales para la investigación biomédica con animales del CIOMS (Council for International Organizations of Medical Sciences) establecida por la UNESCO (United Nations Educational, Scientific and Cultural Organization) y la OMS (Organización Mundial de la Salud) de 1949 y de la Ley 84 de Octubre 27 de 1989 (Estatuto Colombiano de Protección Animal) (10).

Protocolo Anestésico. Todo el procedimiento quirúrgico fue realizado bajo anestesia general, Como premeditación se utilizó Xilacina 10\% (Erma, Colombia) a dosis de $0.5 \mathrm{mg}$ x Kg Vía intravenosa. Como inductor se utilizó una mezcla de Xilacina 10\% (Erma, Colombia) a dosis de $1 \mathrm{mg}$ x Kg + Ketamina 5\% (Over, Colombia) a dosis de $3 \mathrm{mg}$ x kg + Guayacolato de Glicerilo (Erma, Colombia) a dosis de $110 \mathrm{mg}$ x Kg. Como analgésico en el post operatorio se utilizó flunixin de meglumine $5 \%$ (Vecol, Colombia) a dosis de $1.1 \mathrm{mg}$ x Kg y como antibiótico Oxitetraciclina 5\% intravenosa (Erma, Colombia) a dosis de $10 \mathrm{mg} \times \mathrm{kg}$.

\section{RESULTADOS}

Hallazgos quirúrgicos. Durante el procedimiento quirúrgico bajo anestesia general, se evidenció la presencia de abundante tejido granulomatoso, así como la presencia de grandes cantidades de kunkers acumulados en cavitaciones originadas por la invasión del Pythium insidiosum en el tejido granular. Por lo que se decidió retirar el tejido glandular en su totalidad (Figura 3). 


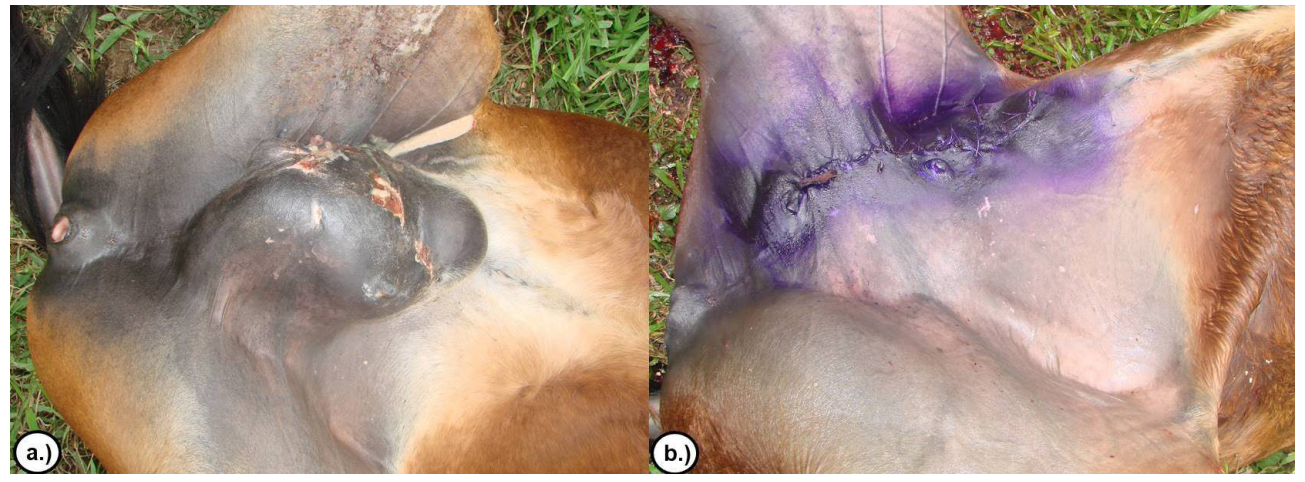

Figura 3. a.) Observe el aumento de tamaño de la glándula mamaria antes de la cirugía. b.) Note la ausencia del tejido glandular y la sutura postquirúrgica en la región mamaria.

Diagnóstico definitivo. La evaluación clínica y la caracterización anatomopatológica de la lesión en la yegua, junto a los resultados histopatológicos confirmaron el diagnóstico de pythiosis cutánea de acuerdo con lo reportado como método diagnóstico de la pythiosis equina $(2,5,6,8,9)$.

Lalesión presentó las cinco manifestaciones clínicas características dela enfermedad, de acuerdo alo reportado porCardona et al $(2,6)$ y Cardona (7), como son la ulceración granulomatosa con superficie irregular, exudación fibrinosanguinolenta, prurito, trayectos fistulosos con salida de material necrótico purulento y caseificado llamados "Kunkers".

Los resultados histopatológicos coincidieron con los informados por Cardona et al $(8,9)$, en la coloración de H\&E, se observó la presencia de marcada infiltración inflamatoria piogranulomatosa, con intensa infiltración de polimorfonucleares (PMN) principalmente eosinófilos y neutrófilos seguido por macrófagos en menor proporción y con distribución difusa, presencia de masas necróticas multifocales y del fenómeno Splendore Hoeppli (SH), lo que corresponde a una dermatitis piogranulomatosa eosinofílica difusa multifocal, así mismo, en la coloración de GMS, se observaron estructuras ramificadas, ocasionalmente septadas, de color marrón oscuro, que algunas veces forman ángulos rectos, lo que corresponden a pseudohifas intralesionales características del oomiceto P. insidiosum.

Seguimiento postoperatorio. Por la poca elasticidad del tejido cutáneo, hubo dehiscencia de sutura en algunos puntos, por lo que se tuvo que manejar la herida con cicatrización por segunda intención, llegando a una recuperación total al día 21 (Figura 4).

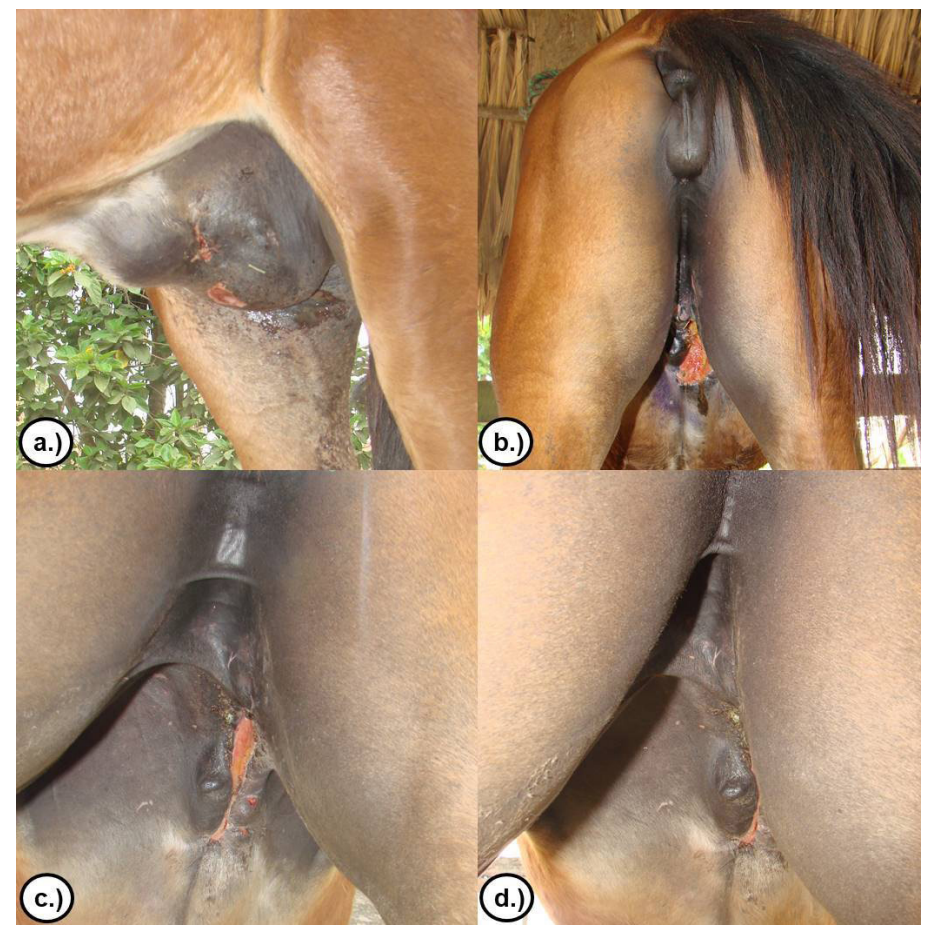

Figura 4. Manejo postquirúrgico por segunda intención. Observe la reducción de la herida con el decorrer del tiempo. a.) Día 0. b.) Día 7. c.) Día 14. d.) Día 21. 


\section{DISCUSIÓN}

Las características iniciales reportadas por lo operarios y su sospecha sobre un accidente ponzoñoso por Loxosceles laeta, queda sin piso científico y clínico, ya que la lesión encontrada no correspondía con los reportado por Cardona, (7) y Cardona et al (11), quienes manifiestan que el loxoscelismo en caballos cursa con dermonecrósis y la presencia de un halo tóxico o progresivo.

Las manifestaciones clínicas encontradas en la lesión, así como las características anatomopatológicas del tejido resecado en la cirugía, sumado a los resultados de las tinciones histopatológicas, confirman el diagnóstico de pythiosis cutánea en la glandula mamaria de una yegua criolla colombiana, de acuerdo a lo reportado por Cardona et al $(2,5,6)$, como método diagnóstico de la pythiosis cutánea equina. De igual forma, las características coinciden con lo reportado por Palmeira et al (12), quienes además del diagnóstico de un caso de mastitis por pythium insidiosum en una yegua que fue remitida al Hospital Veterinário de la Universidad Federal de Campina Grande (Brasil), se realizó igualmente mastectomía total, sin embargo, la yegua fue eutanasiada.

La ubicación en la glándula mamaria, se convierte en el primer reporte en caballos criollos colombianos del Departamento de Córdoba, Colombia; ya que en un estudio de frecuencia de pythiosis cutánea no fue reportada la presentación mamaria de la enfermedad, siendo las partes distales de los miembros la de mayor presentación (5).

Se han realizado diferentes estudios in vitro e in vivo utilizando diferentes antimicóticos tradicionales y de última generación en el tratamiento de la pythiosis equina como ketoconazol, fluconazol, terbinafina, voriconazol e itraconazol, siendo sus resultados pocos satisfactorios e inferiores al 25\%; pudiéndose explicar los resultados insatisfactorios de las drogas antimicóticas por la incapacidad de $P$. insidiosum para producir esteroides en la membrana plasmática, como el ergosterol, que es el componente albo de acción de la mayoría de las drogas antimicóticas, que en los hongos alteran la permeabilidad de la membrana celular provocando la lisis celular del hongo $(13,14)$ En los últimos años, Cardona et al $(6,8,9)$, propusieron el tratamiento para la pythiosis cutanea equina utilizando la acetonida de triamcinolona via intramuscular, con excelente resultados, sin embargo, los propietarios no estuvieron de acuerdo en continuar intentando con el uso de fármacos, debido a la gran cantidad de intentos que habían realizado, por lo que se determinó la opción quirúrgica.

La intervención quirúrgica requiere la remoción de toda el área afectada, con margen de seguridad para evitar las recurrencias, por eso la dificultad de la cirugía en algunas áreas, debido a la complejidad de las estructuras anatómicas comprometidas, principalmente en los miembros, por lo que la mayoría de las veces es asociada al uso de medicamentos como el yoduro de potasio (4).

Se concluye la importancia de conocer la epidemiología de la Pythiosis, siendo relevante la ubicación de casos clínicos a nivel de glándula mamaria en yeguas criollas colombianas expuestas a factores de riesgo como la presencia de heridas en sitios donde puedan tener contacto con aguas contaminadas con Pythium insidiosum.

\section{Conflicto de intereses}

Declaramos no tener conflictos de interés con respecto al trabajo presentado en este informe.

\section{REFERENCIAS}

1. Cardona J, García L, Vergara, O. Pythiosis cutánea equina en Córdoba, Colombia. Reporte de cinco casos. Rev Cientif FCV-LUZ XX 2010; 20(6):590-594. https://produccioncientificaluz.org/index.php/cientifica/article/view/15603

2. Cardona J, Vargas M, Perdomo S. Pythiosis cutánea equina: una revisión. Rev Ces Med Vet Zootec. 2013; 8(1):58-67. https://revistas.ces.edu.co/index.php/mvz/article/view/2837

3. Luis-León J, Pérez R. Pythiosis: Una patología emergente en Venezuela. Salus. 2011; 15(1):79-94. http://ve.scielo. org/scielo.php?pid=S1316-71382011000100009\&script=sci abstract\&tlng=en 
4. Santurio J, Alves S, Pereira D, Argenta J. Pitiose: uma micose emergente. Act Sci Vet. 2006; 34(1):1-14. https://doi. org/10.22456/1679-9216.15060

5. Cardona J, Vargas M, Perdomo S. Frecuencia de pythiosis cutánea en caballos de producción en explotaciones ganaderas de Córdoba, Colombia. Rev Med Vet Zoot. 2014; 61(1):31-43. https://doi.org/10.15446/rfmvz. $\underline{\mathrm{v} 61 \mathrm{n} 1.43882}$

6. Cardona J, Vargas M, Patarroyo J. Pythiosis cutánea en equinos tratados con acetonida de triamcinolona. Parte 1. Caracterización clínica. Rev MVZ Córdoba. 2016; 21(3):5511-5524. https://doi.org/10.21897/rmvz.825

7. Cardona J. Atlas de Dermatología Tropical en Grandes Animales. Fondo Editorial de la Universidad de Córdoba, Universidad de Córdoba: Montería, Colombia; 2018.

8. Cardona J, Vargas M, Patarroyo J. Pythiosis cutánea en equinos tratados con acetonida de triamcinolona. Parte 2. Descripción histológica e histoquímica. Rev MVZ Córdoba. 2017; 22(1):5638-5652. https://doi.org/10.21897/ rmvz.924

9. Cardona J, Vargas M, Patarroyo J. Pythiosis cutánea en equinos tratados con acetonida de triamcinolona. Parte 3. Análisis histomorfométrico. Rev MVZ Córdoba. 2017; 22(2):5881-5898. https://doi.org/10.21897/rmvz.1025

10. Mrad A. Ética en la investigación con modelos animales experimentales. Alternativas y las 3 RS de Russel. Una responsabilidad y un compromiso ético que nos compete a todos. Rev Colom Bioét. 2006; 1(1):163-184. https:// www.redalyc.org/pdf/1892/189217283010.pdf

11. Cardona J, Buitrago J, Martínez N. Caracterización clínica del loxoscelismo dermonecrótico en equinos de Córdoba, Colombia. Rev. CES Med Vet Zoot. 2017; 12(2):123-133. https://revistas.ces.edu.co/index.php/mvz/article/ view $/ 4377$

12. Palmeira R, Lira M, Souza J, Silva T, Miranda-Neto E, Nóbrega N. Mastite em égua causada por Pythium insidiosum. Ciência Veterinária nos Trópicos. 2010; 13(Supl 2):65. http://www.rcvt.org.br/v13 suplemento2 2010/volume13 suplemento2.pdf

13. Argenta J. Atividade in vitro, individual ou em combinação, de voriconazol, itraconazol e terbinafina contra isolados brasileiros de Pythium insidiosum. Act Sci Vet. 2008; 36(3):327-328. https://doi.org/10.22456/1679-9216.17321

14. Cavalheiro A, Maboni G, Azevedo M, Argenta J, Pereira D, Spader T, Alves S, Santurio J. In Vitro Activity of Terbinafine Combined with Caspofungin and Azoles against Pythium insidiosum. Antimicrob Agents Chemothe. 2009; 53(5):2136-2138. https://pubmed.ncbi.nlm.nih.gov/19289531/ 